Ann. Génét. Sél. anim., r971, 3 (3), 225-234.

\title{
ZYTOGENETISCHE BEFUNDE BEI ANGEBORENEN ANOMALIEN DES ZENTRALNERVENSYSTEMS DES RINDES
}

\author{
A. HERZOG und Henni HÖHN \\ Institut für Erbpathologie und Zuchthygiene, \\ Hotmannstrasse Io, D 6300 Giessen (Bundesrepublik Deutschland)
}

\section{ZUSAMMENFASSUNG}

Bei zytogenetischen Untersuchungen von 28 Kälbern mit angeborenen ZNS-Anomalien wurden in 25 Fällen Chromosomenaberrationen beschrieben. Im einzelnen wurden folgende Chromosomenanomalien gefunden :

Zentrische Chromosomenfusion $1 \mathrm{mal}$

Tandem-Fusion-Translokation $1 \mathrm{mal}$

Diploidie-Tetraploidie-Mosaik $23 \mathrm{mal}$

Ob Zusammenhänge zwischen den Chromosomenbefunden und den morphologischen Anomalien bestehen ist nicht zu klären, dazu soll ein grösseres Material untersucht werden.

Während im humangenetischen Schrifttum ZNS-Anomalien verschiedenster Art im Zusammenhang mit Autosomenaberrationen (Trisomien, Polyploidien) diskutiert werden, liegen ähnliche Untersuchungen bei Haustieren unseres Wissens nicht vor, obwohl etwa $25 \%$ der beim Rind vorkommenden angeborenen Entwicklungsstörungen das ZNS betreffen.

\section{MATERIAL UND METHODE}

1. Zur Aufklärung möglicher Zusammenhänge zwischen Chromosomenanomalien und angeborenen morphologischen Anomalien bei Kälbern wurden unter anderem auch von 28 Kälbern mit Anomalien des ZNS und seiner Hüllen Karyotypbestimmungen durchgeführt. In diesem Material fanden sich 25 Fälle mit Chromosomenaberrationen verschiedener Typen (Tabl. 1). In 2 Fällen lagen keine auswertbaren Metaphasen vor und in einem Fall (Anurie) war der Chromosomensatz normal $(2 \mathrm{n}=60)$. 


\section{TABELLE I \\ Autgliederung der Probanden nach Rasse und Geschlecht \\ TABLEAU I}

Répartition des cas selon la race et le sexe

\begin{tabular}{c|l|c}
\hline Anzahl d. Falle & \multicolumn{1}{|c}{ Rasse } & Geschlecht \\
& & \\
\hline & Dt. Schrovzbunt & männl. \\
4 & Dt. Schwarzbunt & weibl. \\
I & Dt. Rotbunt & männl. \\
2 & Dt. Rotbunt & weibl. \\
4 & Dt. Fleckvieh & männl. \\
4 & Dt. Fleckvieh & weibl. \\
I & Dt. Rotvieh & mannl. \\
I & Dt. Rotvieh & meibl. \\
I & Kreuzung & \\
\hline \hline
\end{tabular}

2. Die Metaphaseplatten wurden nach der von BAsRur und Gilmas (1964) angegebenen Méthode aus Leukozytenkulturen des peripheren Blutes sowie aus Kulturen von Knochenmarkszellen (HöHN 1967) gewonnen.

\section{ERGEBNISSE}

$$
\text { Fall I : Totgeborenes Kalb }
$$

\section{Zytogenetischer Betund :}

In 500 ausgezählten Metaphasen beträgt der diploide Chromosomensatz $2 \mathrm{n}=57+\mathrm{XX}$. Auch in 45 tetraploiden Metaphaseplatten finden sich sechs submetazentrische Chromosomen, von denen eines durch besondere Länge der beiden langen Arme auffällt (Abb. I). Je ein Paarling des grössten $\left(A_{1}\right)$ und des kleinsten $\left(\mathrm{E}_{29}\right)$ sind $\mathrm{zu}$ dem grossen submetazentrischen Chromosom verschmolzen (zentrische Fusion).

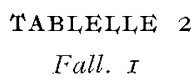

Cas I

\begin{tabular}{c|c|c|c|c}
\hline Rasse & Geschl. & ZNS-Anomalie & Sonstige Anomalien & Kultiviertes Gewebe \\
\hline weibl. & $\begin{array}{l}\text { AnNoLD-CninkI } \\
\text { Anomalie, Spina } \\
\text { bifida aperta, } \\
\text { Hydrocephalus in- } \\
\text { ternus }\end{array}$ & $\begin{array}{c}\text { Neuromyodyspla- } \\
\text { stische Arthrogry- } \\
\text { pose }\end{array}$ & Leukozyten \\
\hline
\end{tabular}




\section{PEV IST}

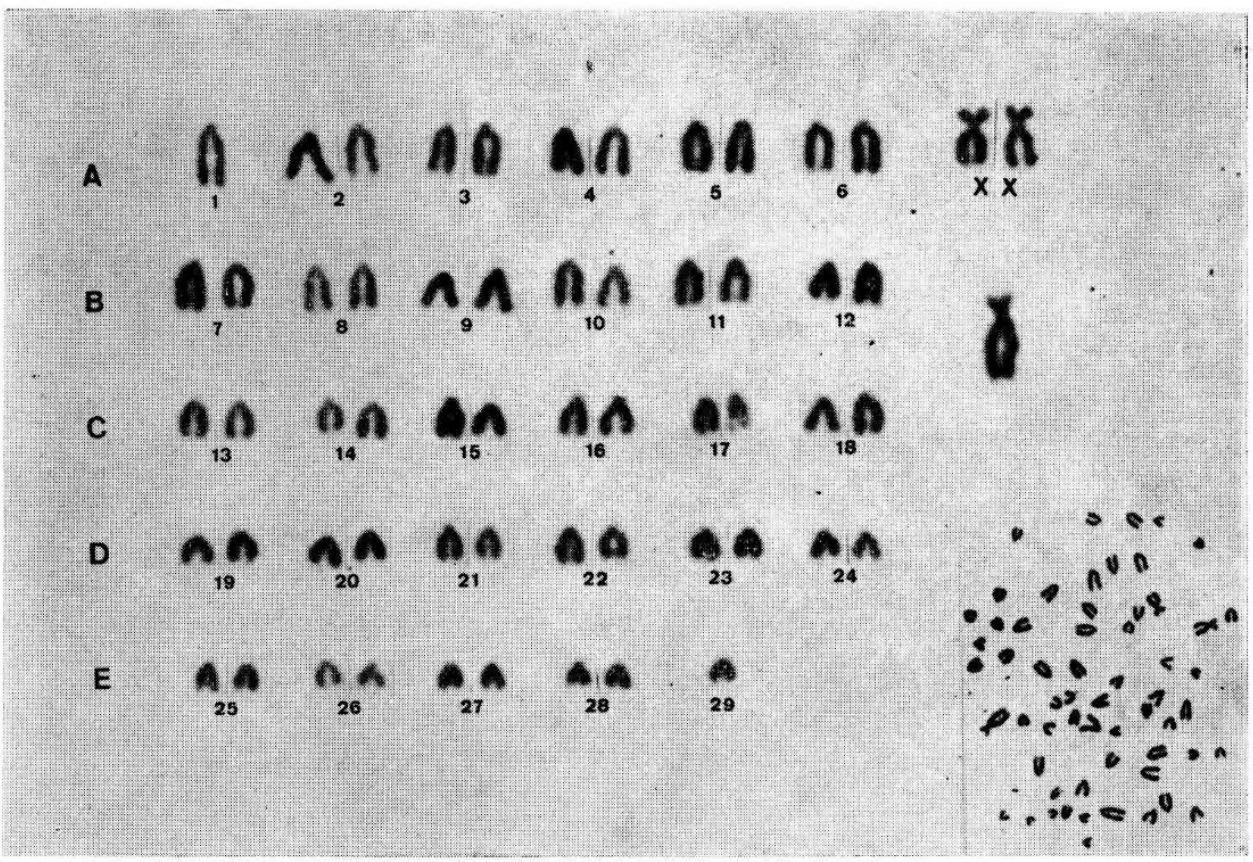

Aвв. I. - Zentromere (I /29) Chromosomenfusion bei einem weiblichen Fleckviehkalb mit Arnold-ChiariAnomalie, Spina bifida aperta, Hydrocephalus internus.

FIG. I. - Fusion centrique I / 29 chez un veau pie rouge présentant l'anomalie d'Arnold-Chiari, une spinabifida ouverte et une hydrocéphalie interne.

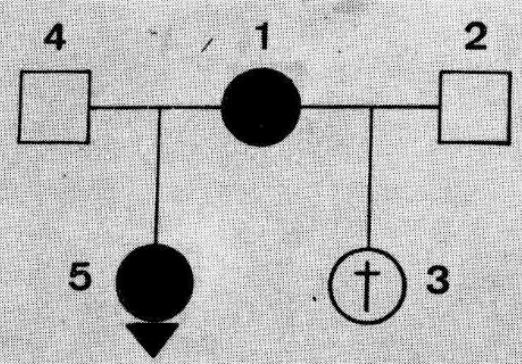

\footnotetext{
= weibl. Träger der Chromosomenfusion (Mutter des Probanden)

= weib1. Trăger der Chromosomenfusion und gleichzeitiger ZNS-Anomalie $\begin{aligned} &(t)= \text { weibl. bereits tote Halbschwester des Probanden mit unbe- } \\ & \text { kanntem Chromosomenbefund }\end{aligned}$

= Vatertiere mit normalem Karyotyp
} 


\section{Zytogenetischer Betund bei Verwandten des Probanden}

Die Mutter besitzt die gleiche Chromosomenanomalie, während der Vater einen normalen Karyotyp hat (Abb. 2). Die Halbschwester des Kalbes konnte zytologisch nicht untersucht werden.

\section{Fall 2 \\ TABELLE 3 \\ Fall 2 \\ TABLEAU 3 \\ Cas 2}

\begin{tabular}{|c|c|c|c|c|}
\hline Rasse & Geschlecht & ZNS-Anomalien & Sonstige Anomalien & Kultiviertes Gewebe \\
\hline $\mathrm{Rb}$ & weibl. & $\begin{array}{l}\text { Hypoplasie d. linken } \\
\text { Grosshirnhemis- } \\
\text { phäre, Spina bi- } \\
\text { fida aperta, seg- } \\
\text { ment. Aplasie d. } \\
\text { Rückenmarks }\end{array}$ & $\begin{array}{l}\text { Anophthalmie-Anu- } \\
\text { rie-Syndrom, } \\
\text { Neuromyodys- } \\
\text { plast. Arthrogry- } \\
\text { pose. }\end{array}$ & Leukozyten \\
\hline
\end{tabular}

\section{Zytogenetischer Befund}

Insgesamt wurden 5II Metaphasen untersucht und mit einer unter gleichen Bedingungen angelegten Kontrolle verglichen. Neben normalen diploiden Metaphasen traten auch solche mit Chromosomenbrïchen und Tandem-Fusion-Translokation auf (Tab. 4).

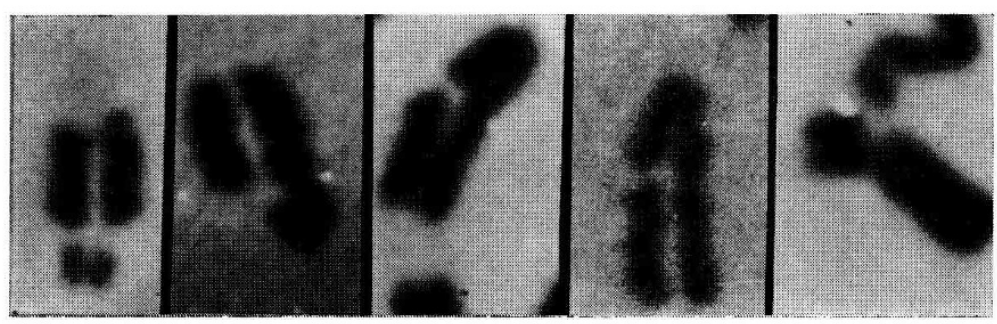

Авв. 3. - Chromosomenbrïche aus 5 verschiedenen Zellen

Fig. 3. - Cassures chromosomiques dans 5 cellules différentes.

Wie Abb. 4 zeigt, handelt es sich bei der Translokation um eine Verlagerung der Chromatiden der Chromosomen des Paares $B_{7}$ nach $A_{1}$, der Briiche der Chromatiden von $B_{7}$ vorausgehen. 
TABELLE 4

Ergebnis der Auswertung von Metaphasen des Probanden 2 und des Kontrollansatzes

TABLEAU 4

Résultats comparés des comptages métaphysiques du cas $n^{0} 2$ avec un témoin

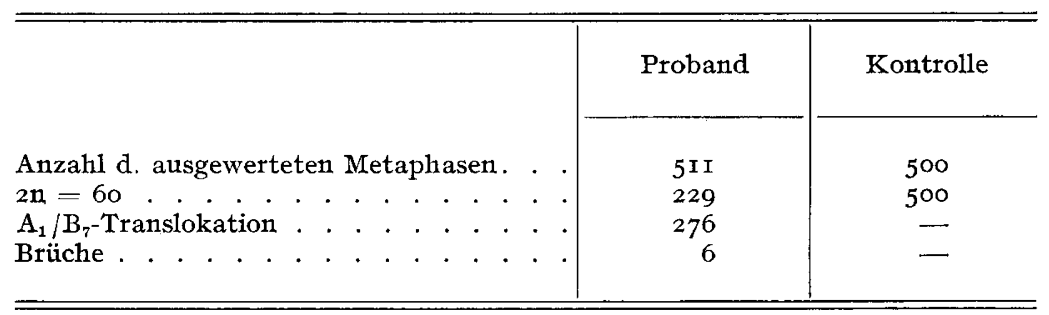

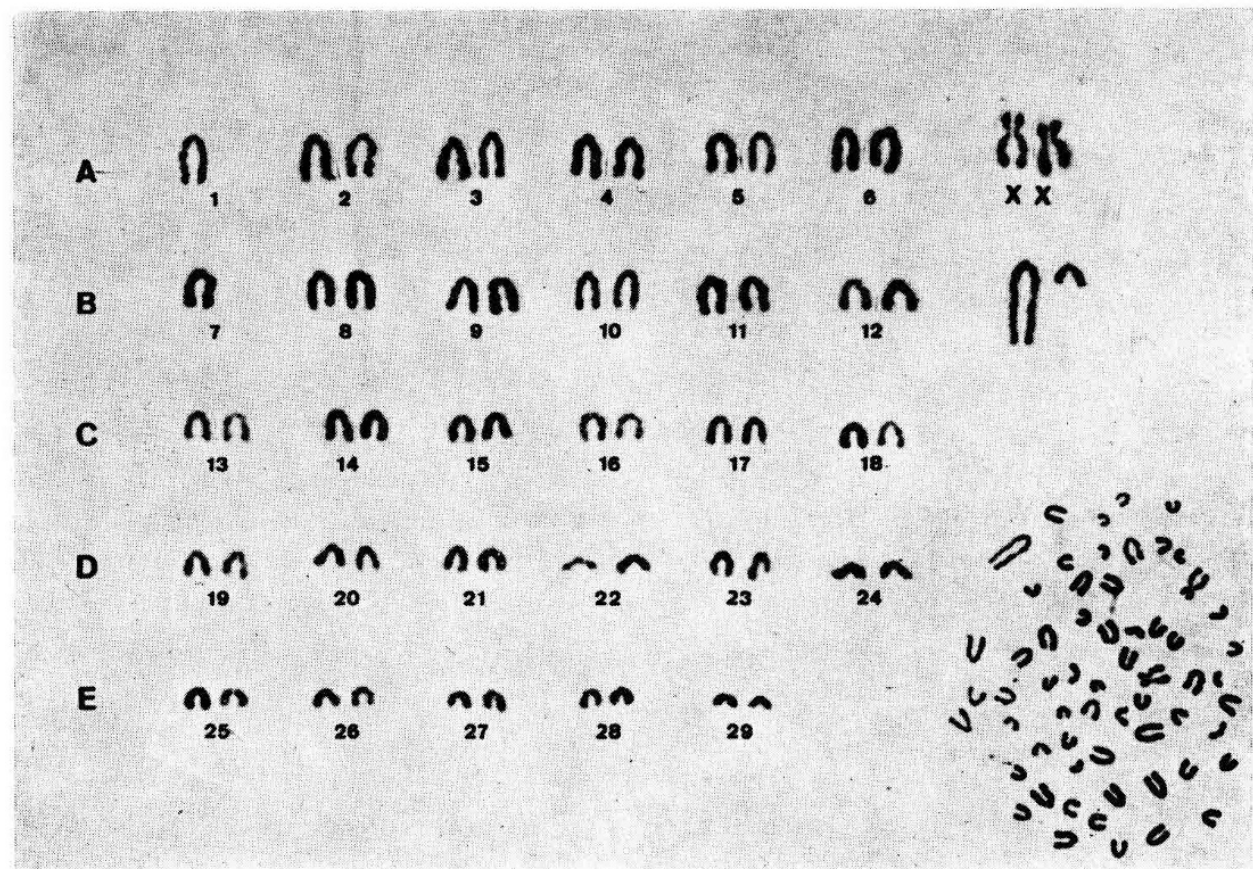

AвB. 4. - Karyotyp des Kalbes mit Tandem-Fusion-Translokation.

FIG. 4. - Caryotype du veau avec fusion translocation en tamdem

\section{Fälle mit Diploidie-Tetraploidie-Mosaik}

Bei dem grössten Teil der zytogenetisch analysierten Kälber mit angeborenen Anomalien des ZNS, seiner Hïllen und der Augen findet sich haüfig ein hoher Anteil von tetraploiden Metaphasen (Abb. 5), der bei gleich behandelten Kontrollen von Kälbern mit anderen Anomalien in keinem Fall vorliegt (Tab. 5). 


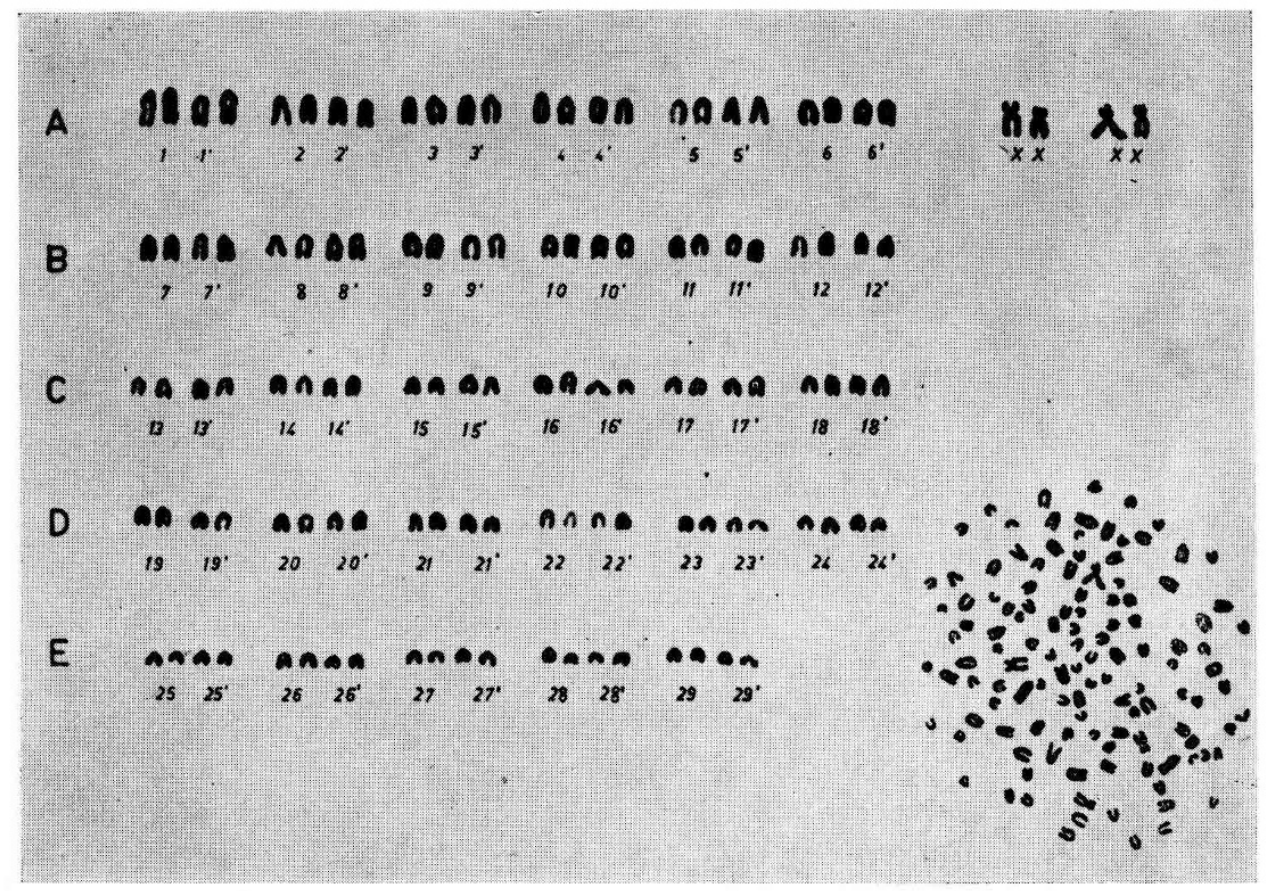

Aвв. 5. - Karyotyp aus einer tetraploiden Zelle

FrG. 5. - Caryotype d'une cellule tetraploïde

Die Art der Anomalien, Rasse, Anzahl der ausgezählten Metaphasen und Anteil der tetraploiden Metaphasen bei den Probanden und Kontrollen sind aus Tabelle $5 \mathrm{zu}$ entnehmen. Die Unterschiede im prozentualen Anteil von tetraploiden Metaphasen bei den Probanden und den Kontrollen sind gesichert $(p<0,00 I)$.

\section{BESPRECHUNG DER ERGEBNISSE}

Die in Fall I beschriebene zentromere Chromosomentransfusion wurde in den letzten Jahren bei Rindern wiederholt im Zusammenhang mit Anomalien, Fertilitätsstörungen, Leukose aber auch bei gesunden Tieren beschrieben (Gustavsson u. Rockborn ig64, Gustaysson i97o, Rieck, Höhn u. Herzog ig68). Aus diesem Grunde muss angenommen werden, dass ein Zusammenhang zwischen ZNS-Anomalie und zentrischer Fusion nicht besteht.

Das gleiche gilt für den Fall 2 mit der Tandem-Fusion-Translokation, die beim Rind erstmals von HANSEN (I970) gefunden und im Zusammenhang mit Fruchtbarkeitsstörungen gebracht wurde. Bei neugeborenen Kindern sollen ähnliche Translokationen auch ZNS-Veränderungen verursachen (BRAY u. MUKHERJEE Ig63).

Die Tandem-Fusion-Translokation ist als spezieller Typ einer chromosomalen 


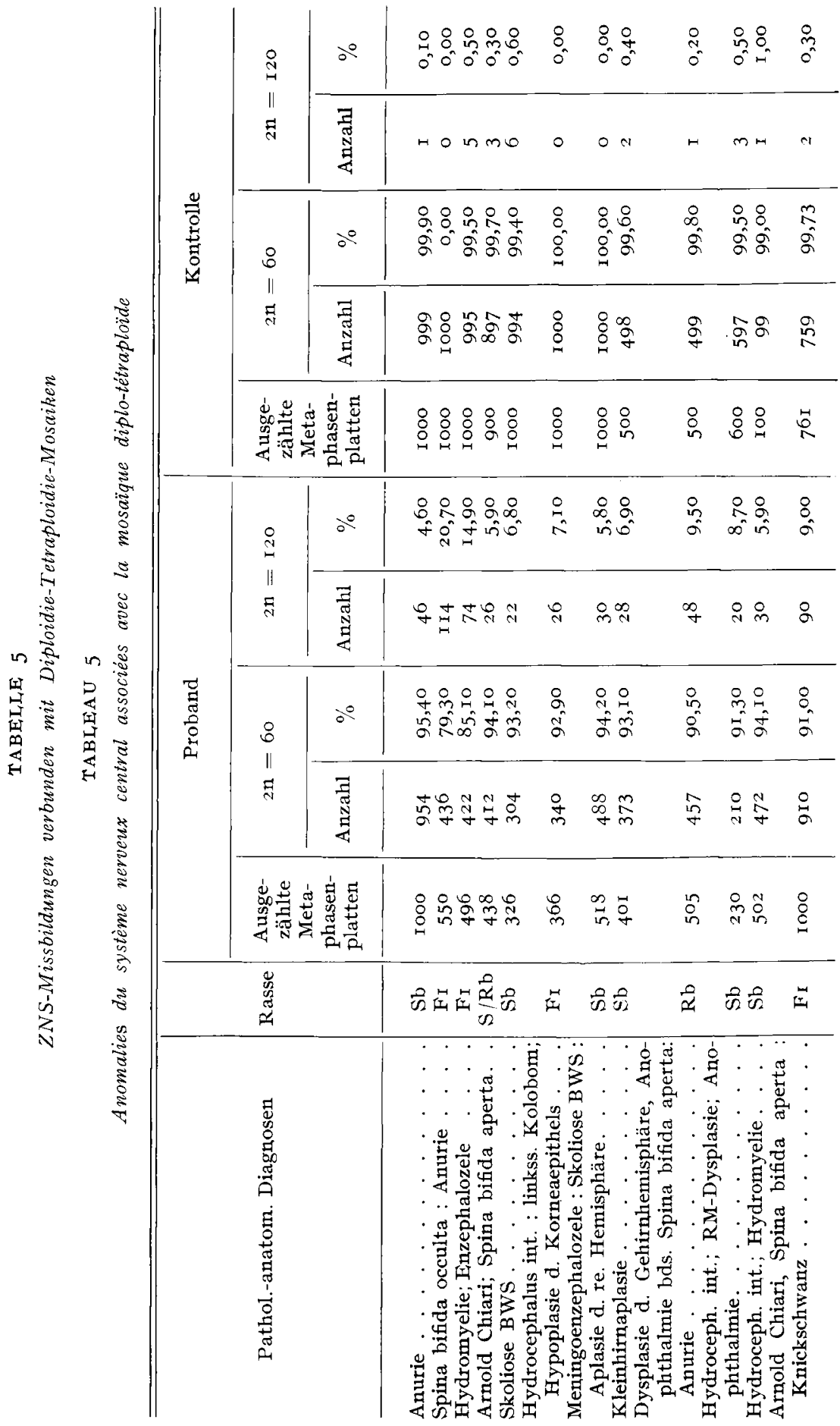




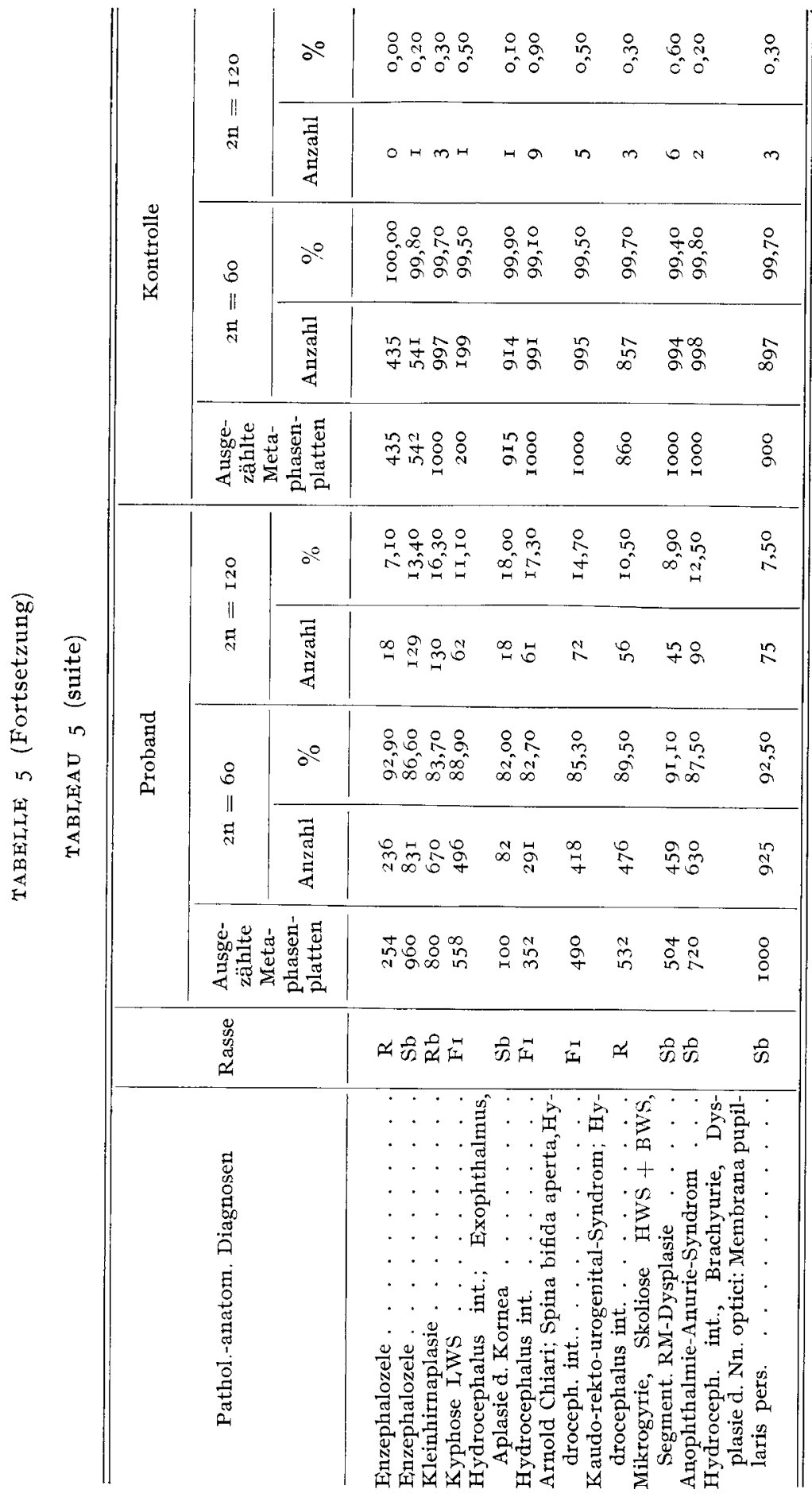


Transposition anzusehen, die durch den Bruch zweier Chromosomen entsteht. In dem einen Chromosom erfolgt der Bruch proximal, um die Zentromeren oder in denselben. Die freien Arme des einen Chromosoms vereinigen sich mit den offenen Enden des anderen Chromosoms. Sind die involvierten Chromosomen akrozentrisch, so verschwinden bei der Mitose die freien Zentromere und die Gesamtzahl der Chromosomen ist um eines reduziert (HANSEN, I970).

Anders scheint es bei den Fällen mit einem Diploidie-Tetraploidie-Mosaik zu sein. Hier könnten gewisse Beziehungen zwischen Chromosomenanomalie und phänotypischer Anomalie bestehen, da auch bei Kindern mit Ploidie-Mosaiken derartig schwerwiegende Anomalien beschrieben wurden (SснмID $u$. VISCHER I967). Obwohl die bei Kälbern mit einem Diploidie-Tetraploidie-Mosaik auftretenden Missbildungen auf den ersten Blick verschieden erscheinen, so liegen sie doch alle in einem bestimmten, ontogenetisch bzw. induktiv voneinander abhängigem Manifestationsareal. Aus diesem Grunde, und auf Grund der Tatsache, dass bei allen anderen von uns zytogenetisch untersuchten Kälbern mit angeborenen Missbildungen (etwa 500 Fälle) ein derartig hoher Anteil an tetraploiden Zellen nicht vorkam, sind Zusammenhänge zwischen der Chromosomenaberration und der phänotypischen Anomalie nicht auszuschliessen. Das vorliegende Material wird noch erweitert, da es zu klein ist um eine endgültige Aussage machen zu können.

$$
\text { Rę̧u pour publication en juin I97I. }
$$

\section{RÉSUMÉ}

ANALYSE CYTOGÉNÉTIQUE DE VEAUX PRÉSENTANT DES ANOMAIIES CONGÉNITALES DU SYSTÈME NERVEUX CENTRAL,

Sur 28 veaux présentant des anomalies du système nerveux central dont le caryotype a été examiné, 25 présentaient des aberrations chromosomiques. On a relevé 1 cas de fusion centrique, un cas de fusion-translocation en tandem et 23 cas de mosaïque ditétraploïde.

Pour préciser d'éventuels rapports entre les défauts chromosomiques observés et les anomalies morphologiques, il faudrait disposer d'un matériel plus important.

\section{SUMMARY \\ CYTOGENETIC ANAIYSIS OF CALVES WITH CONGENITAL, ABNORMALITIES OF CENTRAL, NERVOUS SYSTEM}

The caryotype of 28 calves with central nervous sytem abnormalities have been examined. 25 of them presented chromosome aberrations : centric fusion (1 case), tandem-fusion (1 case), di-tetraploid mosaic ( 23 cases).

To bring out the exact relations between chromosomic defects and morphologic abnormalities a more extensive material is needed.

\section{LITERATUR}

Baskur, P. K.; Gilman J.P. W. I964. A blood culture method for the study of bovine chromosomes. Nature 204, I335-I 337.

Bray, P. F.; MuxherJeE, B., 1963. A chromosome anomaly in an infant with a degenerative disease of the central nervous system. J. Pediat. 62, $230-234$.

Gustavsson, I.; Rockborn, G., I964. Chromosome abnormality in three cases of lymphatic leukaemia in cattle. Nature 203, 990. 
Gustavsson, Y., I970. Economic importance of a translocation in Swedish cattle. I. Europäisches Kolloqium über Zytogenetik (Chromosomenpathologie) in Veterinarmedizin nnd Saugetierkunde, Giessen, 12, u. 13. ro. I970, Giessener Beiträge zur Erbpathologie und Zuchthygiene Sonderheft, I, IO8-II4.

HANSEN, K. M., r970. Tandem-Fusion-Translokation und Unfruchtbarkeit beim Rind. Giessener Beiträge zur Erbpathologie und Zuchthygiene Sonderheft $I$, Ix5-118.

HöHN, Henni, I967. Über Möglichkeiten der Karyotybestimmung post mortem beim Kalb. Giessen, Justus Liebig-Universität, Veterinärmedizinische Fakultät, $D^{\mathrm{r}}$ med. vet.-Diss.

RIECK, G.; HöHN, Henni; HeRzog, A. 1968. Familiäres Vorkommen der zentromeren Chromosomenfusion beim Rind. Zuchthygiene 3, I77-182.

Schmm, W.; VIScher, D., I967. A malformed boy with double aneuploidy and diploid-triploid mosaijism 48, XXYY 71, XXXYY. Cytogenetics 6, I 45-1 55 . 